\title{
Disorder effects on heat transport properties of orientationally disordered crystals
}

\author{
I. V. Sharapova, A. I. Krivchikov, and O. A. Korolyuk \\ B. Verkin Institute for Low Temperature Physics and Engineering of NAS Ukraine, 47 Lenin Ave., Kharkov 61103, Ukraine
}

A. Jezowski

Institute for Low Temperature and Structure Research, Polish Academy of Sciences, PN 1410, 50-950 Wroclaw, Poland

\author{
M. Rovira-Esteva, J. Ll. Tamarit, * L. C. Pardo, and M. D. Ruiz-Martin \\ Grup de Caracterització de Materials, Departament de Física i Enginyieria Nuclear, ETSEIB, Universitat Politècnica de Catalunya, \\ Diagonal 647, Barcelona, Catalonia 08028, Spain
}

\author{
F. J. Bermejo \\ CSIC, Department of Electricity and Electronics, University of the Basque Country, P.O. Box 664, 48080 Bilbao, Spain \\ (Received 16 November 2009; revised manuscript received 8 February 2010; published 30 March 2010)
}

\begin{abstract}
The thermal conductivity $\kappa(T)$ of the orientational glass state of 1,2-difluoro-1,1,2,2-tetrachloroethane $\left(\mathrm{CFCl}_{2}-\mathrm{CFCl}_{2}\right.$, Freon 112) and cyanocyclohexane $\left(\mathrm{C}_{6} \mathrm{H}_{11} \mathrm{CN}\right)$ has been measured under equilibrium pressure within the temperature range $2-100 \mathrm{~K}$. The results show that the soft-potential model is able to account for low-temperature data and, indeed, quantitative agreement of all data considered is found within this realm. The details beyond such temperatures are heavily dependent on chemical details and a large plateau is observed for Freon 112 which is rationalized in terms of resonant scattering of phonons by simple oscillators. Such a view is given additional support by the presence of a strong low-frequency feature in the generalized frequency spectrum of the former material as proven by inelastic neutron-scattering spectroscopy.
\end{abstract}

DOI: 10.1103/PhysRevB.81.094205

PACS number(s): 65.60.+a, 66.70.-f, 61.43.-j, 63.50.-x

\section{INTRODUCTION}

One of the most marked differences in the macroscopic physical properties of crystals and glasses concerns the thermal properties, such as the specific heat $\left(C_{\mathrm{p}}\right)$ and the thermal conductivity $(\kappa) \cdot{ }^{1-5}$ For canonical or structural glasses $(\mathrm{SG})$, that is those truly amorphous structures fully devoid of longrange orientational and translational order, the linear and the quadratic terms in the temperature dependence of the specific heat and thermal conductivity, below $1 \mathrm{~K}$, have been rationalized by means of the tunneling model (TM) ${ }^{6-8}$ Such a model describes the common (also referred to as "universal") features exhibited by the thermal conductivity at $T<1 \mathrm{~K}$ for a large number of SG in terms of the coexistence of localized tunneling modes with sound waves which gives rise a constant density of tunneling states. For temperatures between 1 and $10 \mathrm{~K}$ the anomalous behavior of thermal conductivity that manifests itself by the appearance of a temperatureindependent plateau as well as a concomitant increase in specific heat $\left(C_{\mathrm{p}}\right)$ well above that corresponding to the Debye model, were the subject of conflicting explanations and they have been now rationalized on phenomenological grounds by means of a generalization of the tunneling model known as the soft-potential model (SPM). ${ }^{2-4,8,9}$ It has been shown to be able to account for the plateau of the thermal conductivity on the basis of the assumption of resonant scattering of sound waves from localized low-frequency vibrations. Accordingly, the model can also explain the strong rise of the $C_{\mathrm{p}}$ within the same temperature range. These models have been used to account for the mechanisms governing the poor heat transport properties of SG and indeed the idea of universality of heat transport, i.e., independent of the chemical details arose from these studies. The SPM approach thus provides a de- scription of experimental data for low temperatures in parametric form which needs to be extended if data pertaining higher temperatures are also to be considered. In this latter respect, several relevant attempts have been registered aiming to describe experimental data for temperatures about the plateau as well as above it. For monatomic solids such as silicon, heat transport by phononlike excitations is confined to low temperatures where phonon mean free paths are larger than interatomic spacings. For materials having more complex structures the available experimental data as well as results from recent calculations on model systems, ${ }^{10}$ indicate that heat transfer processes become significantly more complex including energy hopping between localized nonpropagating vibrational modes such as predicted by the model of Allen, ${ }^{11}$ with anharmonicity contributing positively to the thermal conductivity, and its contribution increasing with increasing temperature. Further developments using either simplified models based on an assumption due to Slack $^{12}$ or recent mean-field approaches ${ }^{13}$ cast in terms of fluctuating force constants models have proven their predictive capabilities accounting for thermal data well beyond the plateau in $\kappa(T)$ However, because of their phenomenological nature, none of these models identify the microscopic entities executing tunneling or harmonic motions. To the best of our knowledge, the work carried out by the Cornell group ${ }^{13}$ on the glassy thermal properties of $\mathrm{KBr}: \mathrm{KCN}$ mixed crystals as well as some others inspired by that ${ }^{14,15}$ constitute the known first attempts to progress along such lines.

On a somewhat different vein, recent experimental work on a variety of molecular materials ${ }^{16}$ shows that, even within ordered crystal phases, heat propagation by long-wavelength phonons is bounded to frequencies below a "mobility limit" defined by the condition stating that their mean free path 
should be equal or larger than the phonon wavelength. Above such a limit, heat transport takes place by complex mechanisms which involve interactions between localized and propagating modes. A separation of both long wavelength and diffusive contributions to $\kappa(T)$ can be safely achieved within the orientationally ordered states of some materials which can also be prepared within glassy states ${ }^{17}$ and shows that at temperatures not much higher than the plateau in $\kappa(T)$ heat transport becomes increasingly governed by the diffusive mechanism.

On the other hand, recent studies on heat transport of partially ordered systems have widened the scope of properties considered up to now as an epitome of glassiness, showing that disordered crystals and quasicrystals do conform to the same behavior. ${ }^{18,19}$ In fact, glassy dynamics features may appear even if some translational long-range ordered lattice exists. In particular, some globular-shaped molecules show an intermediate phase between the liquid and the completely ordered low-temperature crystalline phase in which the molecules can rotate more or less freely about the molecular centers of mass which after a time average yield a high space-group symmetry lattice. These phases, referred to as plastic crystals or orientationally disordered (OD) crystals can be supercooled preventing the orientational ordering given rise to an orientational glass (OG) state or glassy crystals. $^{20}$ It has been shown that many characteristic features of SG are shared by the OG as well. ${ }^{21-23}$ Thus, the decoupling between translational and orientational degrees of freedom in OG should make it easier to disentangling the essential properties of the glass state.

In spite of the large number of molecular materials for which the OG state can be reached, data pertaining their thermal properties and, in particular, thermal conductivity at low temperature are scarce. Ethanol is one of the exceptions due to the well-known procedures of obtaining SG and OG for the same compound, thus enabling comparative rationalization of glass properties by means of the SPM. ${ }^{23} \mathrm{~A}$ comparison within the glass state with its closely chemically related compounds as 1-propanol and 2-propanol has highlighted that at low-temperatures SPM accounts for the thermal conductivity behavior and that, in spite of the similarity between both chemical isomers, a huge plateau for 1-propanol extending up to $90 \mathrm{~K}$ (close to the glass transition, $98 \mathrm{~K}$ ) appears, in contrast to the most common plateau found for glasses, as for 2-propanol (up to $10 \mathrm{~K}$ ). ${ }^{22}$ The authors attributed the disparate behavior of both propanols to the differences in the potential energy landscapes due to the existence of additional molecular degrees of freedom in 1-propanol able to effectively hybridize with the sound modes. Thus, the large plateau is explained as a combination between the relaxational scattering (the resonance scattering from quasilocal vibrations) and the scattering from the lowfrequency part of the librational motions appearing in the tail of the density of states $g(\omega)$. Such internal molecular motions for 1-propanol involve low energies (35 K) and thus they would undergo strong coupling to the transverse acoustic phonons producing a complex picture of hybridized modes.

The present report deals with the two aforementioned questions. The first concerns the experimental demonstration of the common scaling of the thermal conductivity according to the SPM for molecular glasses with different types of disorder (SG and $\mathrm{OG}$ ) in the low-temperature region, i.e., below the plateau. In addition to previously published data for the OG state of ethanol (which almost mimics the behavior of its $\mathrm{SG}),{ }^{22}$ the $\mathrm{OG}$ of cyanocyclohexane $\left(\mathrm{C}_{6} \mathrm{H}_{11} \mathrm{CN}\right)$ and 1,2-difluoro-1,1,2,2-tetrachloroethane $\left(\mathrm{CFCl}_{2}-\mathrm{CFCl}_{2}\right)$, named Freon 112, are here given in the frame of some SG formers. The second issue focuses on the origin of the extended conductivity plateau found for Freon 112 and, in particular, concerning the role played in thermal transport by the internal molecular degrees of freedom as a additional scattering source to others intrinsic to disordered systems.

\section{EXPERIMENTAL DETAILS}

Freon 112 is the most fragile OG former known so far. ${ }^{24,25}$ By cooling the high-temperature OD bcc phase, the OG state is achieved at temperatures below $90 \mathrm{~K}$. The existence of two nonequivalent molecular conformations, trans with a $\mathrm{C}_{2 \mathrm{~h}}$ symmetry and gauche with $\mathrm{C}_{2}$ symmetry, has been claimed as the origin of the difficulty to reach the yet unknown low-temperature ordered phase. ${ }^{26}$ Specific-heat measurements have shown the existence of two additional glasslike transitions, at $130 \mathrm{~K}$ and at $60 \mathrm{~K}$, ascribed to the freezing of the trans-gauche relative proportion and to a secondary relaxation the origin of which is still unclear, respectively. ${ }^{27}$ The intriguing properties of Freon 112, especially when compared to its isomer $\left(\mathrm{CCl}_{3}-\mathrm{CF}_{2} \mathrm{Cl}\right.$, Freon 112a) were ascribed to a higher density of minima in the potential-energy landscape caused by the strong transgauche disorder. ${ }^{25}$ As for $\mathrm{CN}$-cyclohexane, the OD fcc phase can be easily supercooled and only annealing or pressure give rise to the emergence of the orientationally ordered phase. Raman studies demonstrated that only one conformation (axial) appears. ${ }^{28}$

The thermal conductivity of different states of disordered crystals Freon 112 and cyanocyclohexane was measured under equilibrium vapor pressure in an experimental setup already described, using the steady-state potentiometric method. ${ }^{29,30}$

OG state was prepared by very fast cooling (above $50 \mathrm{~K} \mathrm{~min}^{-1}$ ) of the OD sample through the glass-transition region to the boiling temperature of liquid $\mathrm{N}_{2}$. The thermal conductivity of the OG state of Freon 112 and cyanocyclohexane was measured at gradually decreasing temperature from 77 to $2 \mathrm{~K}$ and then increasing temperature up to $100 \mathrm{~K}$.

The neutron-scattering experiments were performed on the IN5 direct geometry cold-neutron chopper spectrometer located at the Institut Laue Langevin (Grenoble, France). This instrument offers a large flexibility for wavelength selection and chopper speed which permits considerable optimization of the energy range, energy resolution, momentum transfer, and count rate.

The present measurements were carried out employing an incident wavelength of $5.5 \AA$, which yielded a Gaussian energy resolution of $72 \mu \mathrm{eV}$ (full width at half maximum) at the elastic peak position, as measured using a vanadium rod having the same dimensions as the sample holder. 


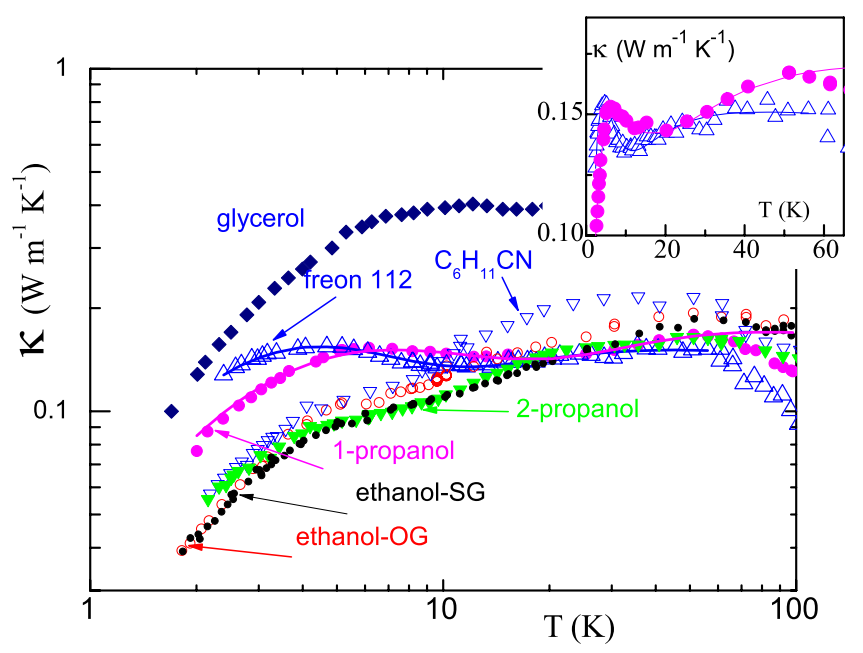

FIG. 1. (Color online) Log-log plot of the thermal conductivity as a function of temperature for SG [full symbols: ethanol, (Ref. 22) black circles; 1-propanol, (Ref. 18) cyan circles; 2-propanol, (Ref. 18) green inverted triangles; and glycerol, (Ref. 3) blue diamonds] and orientational glasses [empty symbols: ethanol, (Ref. 22) red circles; cyanocyclohexane (this work), blue inverted triangles; and Freon 112 (this work), blue triangles]. Solid blue line is the fitted curve using the soft-potential model and the resonant scattering term given in Eq. (5) for Freon 112. Inset shows experimental values and fits for Freon 112 and 1-propanol with a lineal scale for the low-temperature region.

Spectra were recorded for several temperatures ranging from $50 \mathrm{~K}$ up to $140 \mathrm{~K}$ were investigated, which do not reveal strong differences apart from the obvious effects of anharmonicity which are most clearly visible as a broadening of peaks corresponding to the higher frequency excitations with increasing temperature. The data reported here corresponds to the frequency distribution obtained at $70 \mathrm{~K}$.

\section{RESULTS}

Figure 1 shows the thermal conductivity as a function of temperature in a double logarithmic scale for a set of previously published structural glasses $\left[\right.$ glycerol, ${ }^{3}$ 1-propanol, ${ }^{18}$ 2-propanol, ${ }^{18}$ and ethanol-SG (Ref. 22)] and for three orientational glasses, ethanol-OG ${ }^{22}$ Freon 112, and cyanocyclohexane (results of this work). The thermal conductivity exhibits a temperature dependence characteristic for glass materials clearly revealing the strong increase with temperature up to about $3-5 \mathrm{~K}$ followed by a plateau the extent of which heavily depends on the molecular compound involved. Especially relevant is the close similarity between 1-propanol (SG) and Freon 112 (OG), for which the plateau extends up to 70-80 K, i.e., almost up to the glass-transition temperature. A glance to the graph shows the larger conductivity of Freon 112 with respect to 1-propanol in the lowtemperature domain (below $\approx 5 \mathrm{~K}$ ). Such evidence if considered in terms of the SPM suggests that for Freon 112 scattering from low-energy excitations (tunneling states and classical relaxational processes) is stronger that for most of the compiled SG and OG materials (except for glycerol).
The temperature dependence of the thermal conductivity for molecular glasses can be expressed by the standard expression obtained in the Debye approximation for the density of states of sound modes 3,31

$$
\kappa(T)=\frac{k_{B}^{4} T^{3}}{2 \pi^{2} \hbar^{3} v} \int_{0}^{\infty} d x \tau(x) \frac{x^{4} e^{x}}{\left(1-e^{x}\right)^{2}},
$$

where $x=\frac{\hbar \omega}{k_{B} T}, v$ is the velocity of sound averaged over longitudinal and transverse polarizations, and $\tau(x)$ is an effective relaxation time for phonon scattering. The description of thermal conductivity using the SPM is made assuming than in a glass sound modes and soft localized modes of three types interact weakly through the same mechanism. Thus, on the basis of the SPM, an inverse relaxation time $\tau^{-1}(x)$ can be split into three terms describing scattering of sound waves by tunneling states $\left(\tau_{t s}^{-1}\right)$, classical relaxational processes in asymmetric double-well potentials $\left(\tau_{c r p}^{-1}\right)$ and localized vibrations (i.e., soft quasiharmonic vibrations) $\left(\tau_{l v}^{-1}\right)$ (Refs. 2 and 3)

$$
\begin{aligned}
\tau^{-1}(\omega)= & \tau_{t s}^{-1}+\tau_{c r p}^{-1}+\tau_{l v}^{-1}=\bar{C} \pi \omega \tanh \left(\frac{\hbar \omega}{2 k_{B} T}\right) \\
& +\bar{C} \pi \omega\left(\frac{T}{W}\right)^{3 / 4} \ln ^{-1 / 4}\left(\frac{1}{\omega \tau_{0}}\right)+\frac{\bar{C} \pi \omega}{6 \sqrt{2}}\left(\frac{\hbar \omega}{W}\right)^{3},
\end{aligned}
$$

where $\bar{C}$ is a dimensionless constant characterizing the strength of coupling between a sound wave and the soft localized mode without regard for the difference between longitudinal and transverse sound modes. $W$ is the characteristic energy of the quartic potential of the SPM and $\tau_{0}$ is an attempt frequency on the order of $10^{-13} \mathrm{~s}$.

The soft-potential model describing elementary excitations in a wide range of energies $\hbar \omega$ introduces a characteristic energy $W$, which assigns the energy scale in the classification of elementary excitations in the harmonic soft potential. At $\hbar \omega \ll W$ the localized excitations show up as a two-level systems with an almost constant density of states. In the case $\hbar \omega \geq W$ the low-energy modes appear as quasilocal vibrations in a soft single-well potential.

Inserting Eq. (2) in Eq. (1), replacing $\omega$ by its dependence of $x$ and defining a dimensionless variable $z=\frac{k_{B} T}{W}$ (normalized temperature),

$$
\kappa(T)=\frac{2 k_{B}}{\pi}\left(\frac{W}{h}\right)^{2} \frac{1}{\bar{C} v} F(z),
$$

where

$$
F(z)=\int_{0}^{\infty} d x \frac{x^{3} e^{-x}}{\left(1-e^{-x}\right)^{2}} \frac{z^{2}}{1.1 \tanh (x / 2)+0.7 z^{3 / 4}+x^{3} z^{3} / 8}
$$

Function $F(z)$ depends only on the normalized (scaling by $W$ ) temperature $(z)$. Equation (3) is obtained by proceeding from the product $\bar{C} v=C_{l} v_{l}=C_{t} v_{t}\left[v_{j}\right.$ being the velocities of the longitudinal $(j=l)$ and transverse $(j=t)$ sound waves $].{ }^{32}$ 


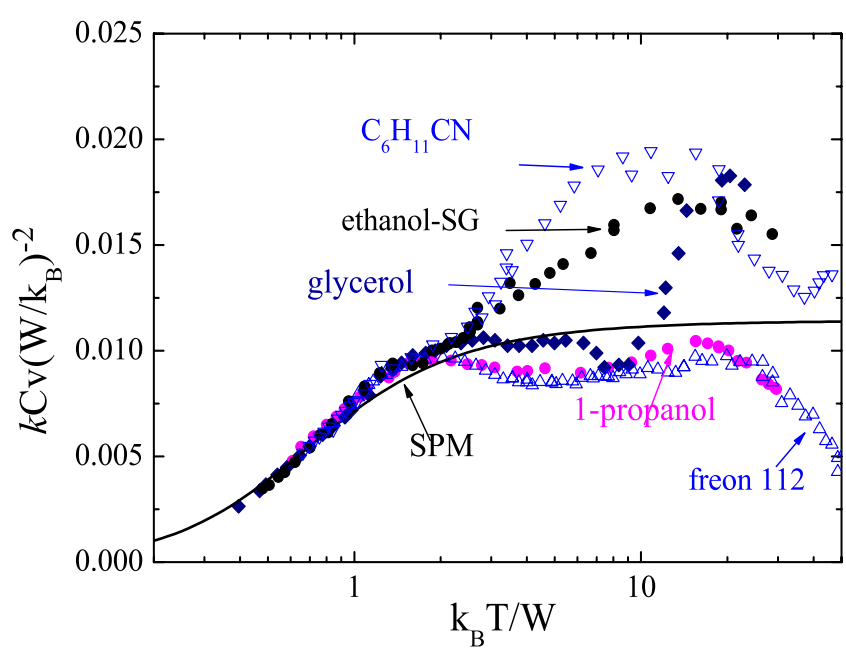

FIG. 2. (Color online) A comparison of low-temperature data for the thermal conductivity of structural and orientational glasses. The dimensionless abscissa is normalized according to $k_{B} T / W$, where $W$ stands from the characteristic energy given by the coefficient of quartic potential term of the SPM. The master curve drawn through the data shows the prediction from the soft-potential model for the same compounds (and the same symbols) as in Fig. 1.

On the grounds of Eq. (3), with only two adjustable parameters of SPM, $\bar{C}$ and $W$, and together with the possibility to evaluate numerically Eq. (4), the temperature dependence of the thermal conductivity should follow the same trends for all kind of glasses (regardless the disorder frozen in). As a result, it means that $\kappa \bar{C} v / W^{2}$ as a function of $\frac{k_{B} T}{W}$ is a glass former independent function for temperatures as high as $k_{B} T / W \leq 2$. Figure 2 provides a direct experimental evidence of the capability of the SPM as an extension of the TM. Table I compiles the most relevant parameters obtained from fitting data to Eq. (3) for the OG state of cyanocyclohexane, Freon 112, and ethanol, while corresponding values for the SG can be found in Refs. 18 and 22. The scaling evidenced in Fig. 2 has two consequences: on the one hand it reassures the validity of the SPM for glass-forming materials irrespective the molecular details for the low-temperature range (in particular for $k_{B} T / W \leq 2$ ), and, on the other it emphasizes the dominant role of the rotational degrees of freedom compared to those giving rise to flow (i.e., mass-transfer) processes. At the microscopic level the quasilocal vibrational modes in orientational glasses can be related to the low-frequency libra-

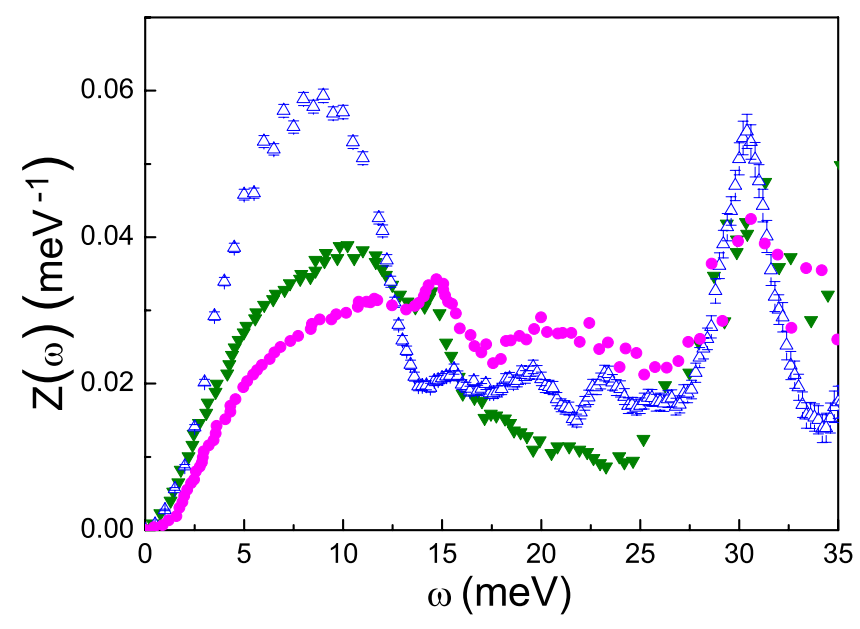

FIG. 3. (Color online) Spectral frequency distribution of the SG 1-propanol and 2-propanol and for Freon 112 (symbols as in Fig. 1)

tion motions of some molecular large-amplitude groups. ${ }^{34}$ The libration motions can be interpreted as quasilocal soft modes interacting with sound modes.

A result that merits some remark shown by Fig. 2 concerns the temperatures where the plateau rises around $T$ $\approx W / k_{B}$, which agrees with the physical meaning of this energy, i.e., the crossover from a regime where phonon scattering by tunneling states and classical relaxators (low-energy vibrations) to another dominated by soft quasiharmonic vibrations. It is also worth noticing the close similarity for the values of the strength parameter $(\bar{C})$ of Freon $112(2.8$ $\left.\times 10^{-4}\right)$ and 1-propanol $\left(3.1 \times 10^{-4}\right)$, both remarkably below those of the OG of ethanol $\left(8.8 \times 10^{-4}\right)$ and SG of 2-propanol $\left(5.4 \times 10^{-4}\right)$, an experimental fact which points out that for temperatures below the plateau scattering from low-energy excitations are weaker in OG of Freon 112 and 1-propanol than in OG of ethanol or SG of 2-propanol. In contrast, as it can be inferred from Fig. 2, SPM overestimates the thermal conductivity for glasses as 1-propanol and Freon 112, for which the nature of the glass is different (SG and OG, respectively). To account for such a deviation at temperatures around $k_{B} T / W \leq 2$ and higher, it was previously proposed for the SG 1-propanol the existence of an additional phonon scattering by molecular modes which become thermally activated about these temperatures. ${ }^{33,35}$ The way to portray such an additional contribution is to add a relaxation time in Eq. (2) which accounts for resonant scattering of phonons by

TABLE I. Parameters of the SPM obtained from the fits of thermal conductivity data (\$). From Ref. 33 (\#). Sound velocity of cyanocyclohexane is unknown.

\begin{tabular}{lcccc}
\hline \hline Chemical & $W / k_{B}(\mathrm{~K})$ & $\bar{C} \times 10^{4}$ & $v\left(\mathrm{~m} \mathrm{~s}^{-1}\right)$ & Ref. \\
\hline Freon 112 (OG) & 2.3 & 2.8 & $1180(\$)$ & This work \\
Cyanocyclohexane (OG) & 3.3 & $\bar{C} \cdot v=0.92 \mathrm{~m} \mathrm{~s}^{-1}(\#)$ & & This work \\
Ethanol (OG) & 4 & 8.8 & 1558 & 22 \\
1-Propanol (SG) & 3.3 & 3.1 & 1727 & 18 \\
2-Propanol (SG) & 3.0 & 5.4 & 1543 & 18 \\
Glycerol (SG) & 4.3 & 2.3 & 2100 & 3 \\
\hline \hline
\end{tabular}


TABLE II. Fitted parameters of the resonant scattering contribution for OG Freon 112 and SG 1-propanol.

\begin{tabular}{lccccc}
\hline \hline Chemical & Energy, $\omega_{0}(\mathrm{~K})$ & $\gamma$ & $F\left(\mathrm{~s} \mathrm{~K}^{-n}\right)$ & $n$ & Ref. \\
\hline Freon 112 (OG) & 25 & 0.001 & $5.510^{-17}$ & 2 & This work \\
1-propanol (SG) & 25 & 0.001 & $3.210^{-17}$ & 1.56 & 18 \\
\hline \hline
\end{tabular}

simple oscillators with an average frequency of $\omega_{0}$

$$
\tau_{\text {res-os }}^{-1}=\frac{F \omega^{2} T^{n}}{\left[1-\frac{\omega^{2}}{\omega_{0}^{2}}\right]^{2}+\gamma\left[\frac{\omega^{2}}{\omega_{0}^{2}}\right]^{4}}
$$

The addition of this contribution to the previous three terms for the effective relaxation time results in the curve (continuous line) is represented in Fig. 1. The approximation is now able to account for the thermal conductivity values through the whole temperature range covered by the plateau. Fitted parameters for the resonant scattering contribution for Freon 112 are summarized in Table II together with those of the SG 1 -propanol as a tool for comparison.

\section{DISCUSSION}

The physical origin of the resonant scattering contribution referred to above is rationalized in terms of coupling between the resonance scattering from quasilocal vibrations and the low-energy molecular librational modes. To the best of our knowledge, the inclusion of such a term provides a microscopic rationale to explain the anomalously mild increase in conductivity with temperature up to about $100 \mathrm{~K}$. Additional data from spectroscopic sources also add weight to the views here pursued. In fact, infrared transmission measurements reveal strong torsional libration of adjacent $\mathrm{CFCl}_{2}$ groups at $\sim 80 \mathrm{~cm}^{-1}(\approx 10 \mathrm{meV})$ and low-energy overdamped librational modes centered at around $40 \mathrm{~cm}^{-1}$ $(\approx 5 \mathrm{meV}) .{ }^{36}$ Such modes have frequencies which overlap those of the high-energy tail of the density of states of acoustic modes which then gives rise to strong mixing of both. This result is consistent with previous studies for which a large plateau appears in the thermal conductivity as 1-propanol ${ }^{18}$ or as those reported for alkali halides. ${ }^{14}$ To add more weight to such a view, the Fig. 3 displays measurements of the neutron-weighted generalized frequency distribution $g(\omega)$ determined from neutron spectroscopic measure- ments. The $g(\omega)$ for Freon 112 shows a remarkably welldefined maximum centered at about $7 \mathrm{meV}$ which on the basis of detailed lattice dynamics calculations carried out for other molecular materials as well as from previous evidence from optical spectroscopy, ${ }^{22}$ can be putatively ascribed to modes having a strong librational character. Such modes due to such hybridization are thus expected to have a partial density of states extending down to frequencies as low as those signaled by the $\omega_{0}$ parameter coming out from the parametric fits (i.e., $25 \mathrm{~K}$ or $2.15 \mathrm{meV}$ ).

In summary, the experimental results we have provided for the thermal conductivity of orientational disordered crystals show the following. (i) The soft-potential model is able to account for the thermal conductivity below $k_{B} T / W \leq 2, W$ being the characteristic energy of anharmonic soft potentials, regardless the chemical particularities and the disorder frozen in to get the glass state, orientational or structural. (ii) The large plateau in thermal conductivity for Freon 112 can be accounted by the existence of resonant scattering of phonons by simple oscillators. Thus, the total conductivity can be decomposed into propagating (phonons) and diffusive-transport contributions, while differences in the component to the conductivity due to diffusive heat transport are mostly attributable to chemical diversity rather than to the existence of an underlying crystalline lattice.

\section{ACKNOWLEDGMENTS}

The authors are sincerely grateful to M. Ramos, H. Schober, E. S. Syrkin, and G. D'Angelo for helpful discussions and interest in this study. This work was financially supported in part by the Spanish Ministry of Science and Innovation (Grants No. FIS2008-00837 and No. MAT200765711-C-4-01) and the Catalan Government (Grant No. 2009SGR-1251) and joint projects of NAS of Ukraine and the Russian Foundation for Fundamental Research (Grant No. N 9-2009).

\footnotetext{
*jose.luis.tamarit@upc.edu

${ }^{1}$ R. O. Pohl, X. Liu, and E. Thompson, Rev. Mod. Phys. 74, 991 (2002).

${ }^{2}$ U. Buchenau, Yu. M. Galperin, V. L. Gurevich, D. A. Parshin, M. A. Ramos, and H. R. Schober, Phys. Rev. B 46, 2798 (1992).

${ }^{3}$ M. A. Ramos and U. Buchenau, Phys. Rev. B 55, 5749 (1997).

${ }^{4}$ F. Bridges and L. Downward, Phys. Rev. B 70, 140201(R) (2004).
}

${ }^{5}$ K. A. Topp, E. J. Thompson, and R. O. Pohl, Phys. Rev. B 60, 898 (1999).

${ }^{6}$ W. A. Phillips, J. Low Temp. Phys. 7, 351 (1972).

${ }^{7}$ P. W. Anderson, B. I. Halperin, and C. M. Varna, Philos. Mag. 25, 1 (1972).

${ }^{8}$ U. Buchenau, J. Phys.: Condens. Matter 13, 7827 (2001).

${ }^{9}$ V. G. Karpov, M. I. Klinger, and F. N. Ignatiev, Solid State Commun. 44, 333 (1982). 
${ }^{10}$ S. Shenogin, A. Bodapati, P. Keblinski, and A. J. H. McGaughey, J. Appl. Phys. 105, 034906 (2009).

${ }^{11}$ P. B. Allen and J. L. Feldman, Phys. Rev. B 48, 12581 (1993).

${ }^{12}$ G. A. Slack, in Solid State Physics, edited by F. Seitz and D. Turnbull (Academic, New York, 1979), Vol. 34, p. 13; D. G. Cahill and R. O. Pohl, Phys. Rev. B 35, 4067 (1987).

${ }^{13}$ W. Schirmacher, Europhys. Lett. 73, 892 (2006).

${ }^{14}$ E. R. Grannan, M. Randeria, and J. P. Sethna, Phys. Rev. B 41, 7799 (1990).

${ }^{15}$ F. J. Bermejo, E. Enciso, A. Criado, J. L. Martínez, and M. Garcia-Hernández, Phys. Rev. B 49, 8689 (1994).

${ }^{16}$ See, for instance, V. A. Konstantinov V. P. Revyakin, and V. V. Sagan, Low Temp. Phys. 35, 577 (2009); V. A. Konstantinov, ibid. 29, 422 (2003).

${ }^{17}$ A. I. Krivchikov, I. V. Sharapova, O. A. Korolyuk, O. O. Romantsova, and F. J. Bermejo, Low Temp. Phys. 35, 891 (2009).

${ }^{18}$ A. I. Krivchikov, A. N. Yushchenko, O. A. Korolyuk, F. J. Bermejo, C. Cabrillo, and M. A. Gonzalez, Phys. Rev. B 77, 024202 (2008).

${ }^{19}$ S. E. Krasavin and V. A. Osipov, J. Phys.: Condens. Matter 13, 1023 (2001).

${ }^{20}$ R. Brand, P. Lunkenheimer, and A. Loidl, J. Chem. Phys. 116, 10386 (2002).

${ }^{21}$ A. Criado, M. Jiménez-Ruiz, C. Cabrillo, F. J. Bermejo, R. Fernández-Perea, H. E. Fischer, and F. R. Trouw, Phys. Rev. B 61, 12082 (2000).

${ }^{22}$ A. I. Krivchikov, A. N. Yushenko, V. G. Manzhelii, O. A. Korolyuk, F. J. Bermejo, R. Fernandez-Perea, C. Cabrillo, and M. A. González, Phys. Rev. B 74, 060201(R) (2006).

${ }^{23}$ M. A. Ramos, S. Vieira, F. J. Bermejo, J. Dawidowski, H. E.
Fischer, H. Schober, M. A. González, C. K. Loong, and D. L. Price, Phys. Rev. Lett. 78, 82 (1997).

${ }^{24}$ C. A. Angell, A. Dworkin, P. Figuiere, A. Fuchs, and H. Szwarc, J. Chim. Phys. Phys.-Chim. Biol. 82, 773 (1985).

${ }^{25}$ L. C. Pardo, P. Lunkenheimer, and A. Loidl, J. Chem. Phys. 124, 124911 (2006).

${ }^{26}$ M. Iwasaki, S. Nagase, and R. Kojima, Bull. Chem. Soc. Jpn. 30, 230 (1957).

${ }^{27}$ K. Kishimoto, H. Suga, and S. Seki, Bull. Chem. Soc. Jpn. 51, 1691 (1978).

${ }^{28}$ T. Woldbaek, A. Berkessel, A. Horn, and P. Klaeboe, Acta Chem. Scand., Ser. A 36a, 719 (1982).

${ }^{29}$ A. I. Krivchikov, V. G. Manzhelii, O. A. Korolyuk, B. Ya. Gorodilov, and O. O. Romantsova, Phys. Chem. Chem. Phys. 7, 728 (2005).

${ }^{30}$ A. I. Krivchikov, B. Ya. Gorodilov, and O. A. Korolyuk, Instrum. Exp. Tech. 48, 417 (2005).

${ }^{31}$ J. J. Freeman and A. C. Anderson, Phys. Rev. B 34, 5684 (1986).

${ }^{32}$ D. A. Parshin, Phys. Rev. B 49, 9400 (1994).

${ }^{33}$ A. Kumar, A. K. Srivastava, and G. S. Varma, Phys. Rev. 178, 1480 (1969).

${ }^{34}$ V. L. Gurevich, D. A. Parshin, and H. R. Schober, Phys. Rev. B 67, 094203 (2003).

${ }^{35}$ R. O. Pohl, Phys. Rev. Lett. 8, 481 (1962).

${ }^{36}$ J. K. Krüger, J. Schreiber, R. Jiménez, K.-P. Bohn, F. Smutný, M. Kubát, J. Petzelt, J. Hrabovské-Bradshaw, S. Kamba, and J. F. Legrand, J. Phys.: Condens. Matter 6, 6947 (1994). 